Int. J. Electrochem. Sci., 12 (2017) $8820-8831$

\title{
Direct Formation of Reduced Graphene Oxide and Graphene Quantum dot Composites by Using Ascorbic Acid as High- Performance Binder-Free Supercapacitor Electrodes
}

\author{
Yongjie Xu, Yukun Feng, Xinyu Li*, Guanghui Hu, Yi Luo, Lang Sun, Tao Tang, \\ Jianfeng Wen, Heng Wang, and Ming L $i^{*}$
}

College of Science \& Ministry-province jointly-constructed cultivation base for state key laboratory of Processing for mom-ferrous metal and featured materials \& Key Lab. of Nonferrous Materials and New Processing Technology, Guilin University of Technology, Guilin 541004, China.

*E-mail: lixinyu5260@163.com, liming928@163.com

Yongjie Xu and Yukun Feng contributed equally to this work.

doi: $10.20964 / 2017.09 .08$

Received: 25 May 2017 / Accepted: 29 June 2017 / Published: 13 August 2017

With unique structural features and hydrophilic functional groups, graphene quantum dots (GQDs) and graphene oxide (GO) are potential materials for supercapacitor electrodes. In this work, GQDs decorated with a GO hybrid and an interconnected nickel network are successfully fabricated and subjected to reduction treatment with ascorbic acid. GO and GQD composites (GO/GQDs) exhibit with different mass ratios of GO to GQDs $(6: 1,3: 1,1: 1)$ are also investigated. The specific capacitance (Csp) of the reduced GO (rGO)/GQD composites is higher than that of rGO. Among the composites with different mass ratios, the rGO/GQD composites with GO:GQDs=3:1 exhibit the highest electrochemical performance because the intercalation of GQDs into GO nanosheets can promote electron transportation and thus reduce the internal resistance and charge transfer resistance of the electrode. Additionally, the GQDs with large specific surface area can enhance the interfacial property among GO. It yields a Csp of $296 \mathrm{~F} \mathrm{~g}^{-1}$ at a current density of $0.5 \mathrm{~A} \mathrm{~g}^{-1}$ and $85 \%$ retention after 3,000 cycles of charge/discharge. Csp remarkably decreases when the mass ratio of GO to GQDs increases to 1:1 possibly because of blocked ion channels from a large amount of GQDs loaded on the GO surface.

Keywords: Graphene oxide quantum dots, Graphene oxide, Reduction, Supercapacitors, Composites

\section{FULL TEXT}

(C) 2017 The Authors. Published by ESG (www.electrochemsci.org). This article is an open access article distributed under the terms and conditions of the Creative Commons Attribution license (http://creativecommons.org/licenses/by/4.0/). 